\title{
El bambú como alternativa de construcción sostenible
}

Bismark Torres ${ }^{1,2 *}$, Mercè Segarra ${ }^{2}$, Luis Bragança ${ }^{3}$

\section{Resumen}

El bambú ha sido y es utilizado por diferentes poblaciones en la construcción de sus viviendas. En América Latina tiene gran presencia: existen 20 géneros y 429 especies de bambúes leñosos que se distribuyen desde México hasta Argentina. En su estado natural es difícil de estandarizar debido a las variaciones que presenta a lo largo de su estructura, tanto en sus propiedades físicas como mecánicas, lo que ha dificultado su incursión masiva en la industria de la construcción. La elaboración de paneles bambú es una alternativa al problema de estandarización; no obstante, esta técnica representa la pérdida de las propiedades estructurales naturales del bambú. A pesar de ello el bambú sigue siendo una alternativa de construcción sostenible. En este trabajo se hace una revisión de la investigación publicada sobre el uso del bambú como una alternativa de construcción sostenible, desde los puntos de vista de su capacidad para secuestrar carbono, la reducción del impacto ambiental que aporta a los sistemas constructivos, sus propiedades estructurales y sus posibles aplicaciones arquitectónicas.

Palabras clave: bambú; construcción sostenible; secuestro de carbono; impacto ambiental.

\section{Abstract}

Bamboo has been used by different populations in the construction of their dwellings. In Latin America it has a great presence: there are 20 genres and 429 species of bamboos that are distributed from Mexico to Argentina. In its natural state it is diffcult to standardize due to the variations that occur throughout its structure, both in its physical and mechanical properties, which has hindered its massive incursion

\footnotetext{
${ }^{1}$ Departamento de Investigación, Facultad de Arquitectura y Urbanismo, Universidad de Guayaquil, Guayaquil, Ecuador. ${ }^{2}$ Centro DIOPMA, Departamento de Ciencia de Materiales y Química Física, Facultad de Química, Universitat de Barcelona, Barcelona, España.

${ }^{3}$ Centro CTAC, Departamento de Engenharia Civil, Universidade do Minho, Guimaraes, Portugal.

*bismark.torresr@ug.edu.ec
} 
in the construction industry. The elaboration of bamboo panels is an alternative to the problem of standardization. However, this technique may represent the loss of the natural properties of bamboo. Despite this, bamboo remains an alternative for sustainable construction. This paper reviews the published research on the use of bamboo as a sustainable construction alternative, from the point of view of its capacity to sequester carbon, its environmental impact on construction systems, its structural properties and its possible architectural applications.

Keywords: bamboo; sustainable construction; carbon sequestration; environmental impact.

\section{Introducción}

Existe una necesidad urgente de establecer un nuevo paradigma para promover la conservación y la sostenibilidad ambiental. Para conseguirlo se necesita considerar en los productos la disponibilidad suficiente, capacidad de renovación, características físico-mecánicas adecuadas, eficiencia de costos, fácil acceso, producción simple, tecnología local adaptada a las condiciones locales y durabilidad. El bambú [1][2] se presenta como una alternativa a este paradigma, debido a que se encuentra en todas las regiones del mundo y sus 1.600 especies están repartidas en 33 países en un área aproximada de 31,5 millones de hectáreas. Presentan un rápido crecimiento y propagación sin que haya necesidad de replantarlo después del aprovechamiento adecuado, lo que representa ventajas productivas, económicas y ambientales. Se lo utiliza en ali- mentación, construcción, artesanía, papel, paneles, tableros, laminados, suelos, techos, tejidos, aceite, gas, carbón vegetal, entre otros. En América Latina tiene gran presencia: existen 20 géneros y 429 especies de bambúes leñosos que se distribuyen desde México hasta Argentina. Dentro del género guadua en América se destaca la guadua angustifolia Kunth, nativa de Colombia, Ecuador y Perú, por sus excelentes propiedades físicas y mecánicas, que tiene una tasa de crecimiento de $10 \mathrm{~cm}$ por día, llegando a alcanzar los $30 \mathrm{~m}$ de altura y $25 \mathrm{~cm}$ de diámetro [3][4][5]

Frente al calentamiento global, la crisis energética y las tensiones socio-ambientales, el uso de energías renovables, los materiales y tecnologías sostenibles no convencionales se convierten en un gran aliado para minimizar el impacto de los convencionales y fomentar cambios en las actitudes de los profesionales involucrados [6]. La industria del bambú actualmente está en auge en Asia, y se extiende rápidamente a otros continentes como África y América [2]

\section{El bambú y el secuestro de carbono}

El carbono atmosférico (CO2) es absorbido por los árboles mediante la fotosíntesis y es almacenado en forma de materia orgánica (biomasa). E1 CO2 regresa a la atmósfera mediante la respiración de los árboles y por descomposición de la materia orgánica.

El bambú es considerado un material ecológico debido a la capacidad que tiene para secuestrar carbono a altas tasas en 
función de su rápido crecimiento y producción de biomasa, está considerado dentro del grupo de plantas $\mathrm{C} 4$. Se regenera por sí solo a partir de rizomas, no necesita ser replantado. Puede crecer en terrenos no aptos para otros cultivos, lo que lo convierte en un complemento conveniente y útil para sistemas agrícolas y agroforestales diversificados, grandes y pequeños [1] [4] $[7][8][4][9]$.

\subsection{Fijación del carbono en los bosques de Bambú}

La fijación de carbono en los bosques de bambú ha sido objeto de algunas investigaciones. Se observa que la cantidad de biomasa sobre el suelo es bastante similar en la mayoría de especies estudiadas: 121,51 th ha $\mathrm{a}^{-1}$ Bambusa cacharensis, Bambusa vulgaris y Bambusa balcooa [10], 137,9 tha-1 Phyllostachys pubescens [11], 143 thha-1 Bambusa blumeana[12], $162 \mathrm{t} \cdot \mathrm{ha}-1$ para Chusquea culeou [13] y 135,8 tha-1 para Phyllostachys bambusoides [14]. E1 mayor aporte de biomasa se da en el culmo seguido por la rama y la hoja [5][10][11]. En la especie Phyllostachys pubescens la tasa de crecimiento de la biomasa por encima del suelo es de 3,0 t C ha-1año-1.

Para las especies Bambusa cacharensis, Bambusa vulgaris y Bambusa balcooa [10] el stock bruto de carbono en la plantación se estimó en 120,75 tha-1 de los cuales el $51 \%$ corresponde al almacenamiento en la biomasa sobre el suelo (culmo 87\%, rama $10 \%$, hoja $3 \%$ ), el $2 \%$ en la masa del piso de la camada y el $47 \%$ en el suelo hasta $30 \mathrm{~cm}$ de profundidad. Para la especie Phyllosta- chys pubescens el stock total de carbono en la reserva fue de 78,6 tha-1 en el cuerpo de la planta y 101,2 tha-1 en el sistema del suelo [11]

La fijación de carbono estimada Guadúa angustifolia, para un período de crecimiento de 6 años, es de 54,3 t, donde 10,8 $\mathrm{t}(19,9 \%)$ de la fijación de $\mathrm{CO} 2$ corresponde al rizoma y 43,5 t $(80,1 \%)$ a la parte aérea del grupo; en las primeras etapas de crecimiento, la contribución del rizoma y el culmo alcanza el 50\% de la biomasa total y, seis años después, la contribución del rizoma y el culmo a la biomasa total alcanza el 90\% [5].

E1 suelo de bambú tiene implicaciones importantes en la manipulación del $\mathrm{CO} 2$ atmosférico, al igual que el suelo de los bosques tropicales [10].

\subsection{Almacenamiento de carbono en productos}

La contribución anual de carbono no es uniforme: las generaciones de menor edad aportan una mayor proporción de biomasa que las de mayor edad [1]. La cosecha de bambú de 4 a 5 años fomenta un manejo cuidadoso de las plantaciones [15], lo que hace que el bambú sea más productivo. El hecho de usar el bambú con elevados porcentajes de biomasa en soluciones constructivas duraderas, representa un mayor almacenamiento de carbono como biomasa a largo plazo. El carbono secuestrado y almacenado en la biomasa del bambú recolectado sólo será liberado al ambiente por un proceso de combustión o biodegradación. El uso de soluciones de 
bambú industrializadas en programas de vivienda en Filipinas [15] mostró efectos positivos sobre el medio ambiente al capturar y evitar más de 108 tCO2 de emisiones durante 130 años. Las innovaciones recientes en el procesamiento y el desarrollo de productos han aumentado la porción de bambú convertido en productos duraderos como materiales de construcción, tableros para pisos, productos de paneles y muebles. Esto refuerza la contribución de los productos de bambú al secuestro de carbono a largo plazo [9].

\subsection{E1 bambú y otras especies madereras}

No se encontró mucha información respecto a la capacidad de secuestro de carbono del bambú frente a otras especies madereras. Sin embargo, un estudio donde se compara el bambú Phyllostachys, el pino y el eucalipto revela que el bambú almacena cerca del $45 \%$ del carbono en su biomasa, valor similar al de estas otras especies; pero si se incluye la tasa de crecimiento y la productividad anual almacena el $41 \%$ y el 21\% más que el pino y el eucalipto respectivamente [16]. También su capacidad de secuestro es mayor cuando lo comparan con el abeto de China [17].

La International Network for Bamboo and Rattan (INBAR) hace una extensa revisión de la literatura sobre la producción de carbono y biomasa de los bosques de plantación en China, y concluye que el bambú parece ser una opción viable para el secuestro de carbono dentro de la silvicultura [18].

\section{El impacto ambiental del bambú en los sistemas constructivos}

Evaluaciones del ciclo de vida de materiales de construcción basados en bambú concluyen que los impactos ambientales de estos materiales crecen con una mayor industrialización [19][20]. Los insumos relacionados con la recolección y el transporte de bambú y la resina utilizada en el producto tienen una contribución extremadamente limitada al impacto ambiental, mientras que la naturaleza y la cantidad de energía utilizada en el proceso de producción son parámetros críticos que contribuyen de mayor manera a la variabilidad de los resultados [19]. Sin embargo, en un estudio de paneles prensados a base de Guadua Angustifolia Kunth (GAK), en el análisis del ciclo de vida determinaron que los principales contribuyentes a la huella de carbono son la producción de los productos químicos del pegamento (urea formaldehido y acetato de vinilo) y el transporte de las guaduas desde la granja hasta la planta de fabricación [20].

Una investigación [21] donde se compara el impacto ambiental de los materiales de bambú producidos en China y enviados a Europa occidental para su comercialización, con la madera, concluyen que: el procesamiento de productos de bambú y el transporte consumen la mayor cantidad de energía; los productos de bambú tienen menor impacto ambiental y mayor rendimiento que la madera dura tropical; el tallo de bambú y los productos de bambú de segundo grado, como los defibra de densidad media MDF y aglomerados, son soluciones sostenibles para aplicaciones locales; sin 
embargo, tienen mayor impacto ambiental que los productos europeos de segundo grado de madera blanda europea. Un hallazgo sorprendente de la evaluación es que la resina utilizada para procesar productos de bambú industrial modernos contribuye poco a la huella de carbono o al costo ecológico. Sin embargo, hay espacio para mejorar utilizando resinas que contengan menos formaldehido.

\section{Propiedades estructurales del bambú.}

E1 bambú [22][23] es un material compuesto que está reforzado longitudinalmente por fibras de celulosa; a lo largo de su estructura presenta variaciones de volumen respecto al radio. La distribución de las fibras del bambú aumenta desde el interior hacia el exterior lo que les permite actuar como un refuerzo similar al de barras de acero en concreto. La evaluación de la integridad estructural del bambú con fines de construcción es esencial para garantizar que su uso se alinee con las aplicaciones requeridas.

E1 bambú responde muy bien a tensiones paralelas a la fibra. Sin embargo, a tensiones perpendiculares su respuesta es débil debido a la lignina, aunque esta característica junto a la del peso y su forma hueca representa una ventaja en caso de un terremoto [22][23][24]. El rendimiento mecánico del bambú depende mucho de la edad. El bambú joven tiene una mayor resistencia, rigidez elástica y resistencia a la fractura en comparación con su contraparte más antigua. La excelente toleran- cia al daño del bambú puede atribuirse a la interacción y despliegue concurrente de la deflexión de grieta y el puente de grieta como los principales procesos de disipación de energía [25]. El bambú tiene mejor resistencia a la compresión que algunos otros materiales de construcción, como la madera, el hormigón y el ladrillo, y la resistencia a la tracción es comparable a la del acero [26]. También se compara favorablemente con muchas especies de madera en términos de su resistencia a la tracción, flexibilidad y dureza [9][22][23][27][28].

La unión de las cañas representa un desafío; sin embargo, la elaboración de juntas, es una propuesta viable para mejorar las conexiones estructurales de los elementos de bambú [24]

Las fibras naturales of recen ciertas ventajas en comparación con las sintéticas, ya que son renovables, biodegradables, menos abrasivas, y presentan una menor demanda energética para su producción. Un estudio [29] donde utilizan fibras bambú para la obtención de materiales compuestos de polipropileno, sin y con agente acoplante (anhídrido maleico de polipropileno), concluyen que la resistencia máxima a tensión y flexión para la mayoría de los materiales compuestos fue inversamente proporcional al aumento de partículas de bambú y directamente proporcional al agente acoplante, sin superar al plástico; mientras que el módulo de elasticidad en ambas propiedades aumenta en forma directa con el contenido de partículas y el agente acoplante por encima del plástico. La resistencia al impacto para los materia- 
les compuestos es directamente proporcional, cuando se agrega agente acoplante.

Los productos de bambú diseñados resultan del procesamiento del culmo de bambú crudo en un material compuesto laminado o prensado, y son similares a los productos de madera laminada encolada. Estos productos permiten que el material se use en secciones estandarizadas y tienen menos variabilidad inherente que el material natural.

Respecto a las propiedades mecánicas de tensión y compresión, el bambú prensado tiene resistencias ligeramente más altas que el bambú laminado, mientras que en la propiedad de corte paralelo a la fibra su resistencia es menor [30]. Sus propiedades mecánicas son comparables a otros materiales estructurales como la madera y el bambú en bruto. Una ventaja del bambú laminado es su relación de resistencia a la flexión y densidad. La resistencia a la flexión del bambú diseñado es superior al del bambú natural y más baja que la de los compuestos de polímeros reforzados con fibra [28][31][32]. La densidad [33][34] [35] tiene una influencia significativa en las propiedades mecánicas de los paneles de bambú, así como también en la absorción de agua.

Tanto el bambú prensado como el laminado exhiben el comportamiento anisotrópico típico del bambú natural y similar al de los compuestos reforzados con fibra [23][36].

Las cargas sísmicas son proporcionales al peso de la estructura y son cíclicas, lo que puede causar fallas de fatiga en las conexio- nes. Históricamente los edificios tradicionales de bambú y quincha / bahareque han tenido un buen desempeño en terremotos por dos razones claves: su ligereza (alta resistencia en relación a su peso) y su ductilidad (esencialmente la capacidad de absorber energía) en las conexiones y uniones/ empalmes, especialmente cuando se usan clavos [37].

En la India, realizaron un estudio utilizando bambú como reemplazo del acero donde las propiedades mecánicas del concreto reforzado con bambú [38] se presentaron en términos de resistencia a la compresión, resistencia a la tracción dividida y resistencia a la flexión. Los resultados muestran con satisfacción las posibilidades de utilizar el refuerzo de bambú como reemplazo del refuerzo de acero en estructuras de concreto pueden ser factibles en términos de construcción ecológica de bajo costo. Otro factor como la resistencia del concreto reforzado con bambú también está aumentando con la edad. En el campo de la construcción verde, el uso del bambú como reemplazo de acero, donde la disponibilidad de material de acero es baja y el costo es alto, puede ser la mejor solución. También se sugiere que la participación del bambú en la construcción verde puede disminuir el uso del acero y puede convertirse en una de las mejores soluciones con recursos limitados en áreas remotas para personas necesitadas. Sin embargo, debido a la tendencia del bambú a absorber agua, la unión entre el bambú y el concreto se considera el mayor problema [39]. 
La falta de códigos y estándares de construcción apropiados es una barrera para los ingenieros y arquitectos en el uso del material. La estandarización de los productos de bambú estructural refleja el creciente interés de la sociedad y los responsables políticos y ofrece una nueva apertura para el desarrollo industrial sostenible. El surgimiento de una codificación completa de los productos de bambú estructural puede ser inevitable en un futuro cercano,

Aunque las propiedades mecánicas del bambú están por encima de la media, el bambú es muy susceptible [37][40] al fuego, la humedad, las termitas y escarabajos, por lo que necesita ser tratado para protegerse contra ellos y mantener sus propiedades mecánicas.

\section{Aplicaciones Arquitectónicas (soluciones constructivas).}

E1 bambú es una de las plantas más primitivas del mundo. Debido a sus excelentes propiedades mecánicas se ha utilizado continuamente en el campo de la construcción en aplicaciones de paredes, techos, pisos, puentes, andamios, entre otras [26]. Se estima que más de mil millones de personas en todo el mundo viven en casas de bambú o utilizan este material como elemento principal para la estructura, el revestimiento exterior o la techumbre [1]. A pesar de la larga tradición de construcción con bambú, el material no se utiliza con frecuencia en construcciones modernas; la aplicación del bambú en el diseño aún se está desarrollando y se es- peran nuevas aplicaciones [31][39] que continúen incrementando la porción de bambú convertido en productos duraderos.

E1 bambú es considerado un material versátil debido a su alta relación resistencia-peso, facilidad de trabajo y disponibilidad; dependiendo de su situación y aplicación puede remplazar al concreto, la madera o el acero [39][41][38]. Una mayor participación del bambú disminuiría el uso del acero y, con ello lo que su uso significa en términos de impacto ambiental. Esta también podría ser la mejor solución, en lugares donde la disponibilidad del acero sea baja y el costo alto.

Los productos de bambú diseñados resultan del procesamiento del culmo de bambú en un material compuesto laminado, similar a los productos de madera laminada encolada. Estos productos permiten que el material se use en secciones estandarizadas y tienen menos variabilidad inherente que el material natural [31]. El sustento del bambú para aplicaciones de construcción ofrece beneficios, tales como prefabricación, ensamblaje simple, reemplazo simple de partes estructurales y, además, los elementos de bambú podrían ser fácilmente desmantelados y reutilizados para otra aplicación [26].

Hay estudios prometedores, donde, dependiendo de sus propiedades higiénicas, térmicas y mecánicas, los tableros de fibra de bambú se pueden usar como materiales de aislamiento de edificios en paredes, techos o en espacios abuhardillados; así como también pueden participar en el control de humedad interior [42]. El 
ahorro de energía en la construcción por transferencia de calor a través de paredes externas es otro tema importante en la selección de materiales. Las estructuras de paredes de acero y bambú tienen un mejor rendimiento energético que las estructuras de pared comunes [43], lo que indica un gran potencial para el uso de estas paredes en edificios residenciales en las zonas cálidas de verano e invierno frío; sin embargo, el principal inconveniente de la estructura de acero y bambú es que su rendimiento térmico interior durante las horas más calurosas del verano es más pobre que las paredes convencionales. No obstante, todavía falta mucho por investigar sobre estos temas.

En muchas regiones en desarrollo, existe un déficit de vivienda digna, de bajo costo, sostenible de construcción rápida y fácil, que resista a las tensiones producidas por el viento y/o terremotos [38][37]. Esta realidad ha motivado a autoridades e investigadores a pensar en el bambú como una alternativa de solución sostenible a la problemática planteada; lo que los ha llevado a seguir investigando en las bondades y usos del bambú, así como también a la creación de normas de construcción con bambú [44][45][46][47][9][1]. La Organización Internacional de Normalización (ISO) en 2004 publicó tres normas sobre la construcción de bambú (ISO, 2004a, 2004b, 2004c). Países como China, Colombia, Ecuador, India y Perú han tomado la iniciativa en la creación de un marco para los códigos de construcción [44]. Los resultados en el desarrollo de un método de diseño de vivienda, usando paredes de paneles de bambú contrachapado con base en conexiones entre paredes, muestran que el método seleccionado podría ser implementado en regiones propensas a terremotos y vientos de magnitud considerable [48]. Poco a poco el bambú va recibiendo un mayor reconocimiento como material de construcción. Los pocos programas de construcción de casas de bambú a gran escala, para grupos de bajos recursos económicos, han tenido gran éxito respecto a su objetivo principal, el de proporcionar refugio a las familias pobres [46]. La Universidad Nacional de Colombia, en Bogotá, en el 2008, llevó a cabo el proyecto de "Diseño y construcción de viviendas con elementos estructurales de bambú laminado". El mismo consistía en implementar la producción de elementos estructurales en bambú laminado, elaborar el diseño arquitectónico y estructural y, la construcción de un prototipo de vivienda [49].

E1 bambú ofrece una variedad de opciones de construcción, desde costos muy bajos hasta costos elevados, y, por lo tanto, tiene una gama más amplia de grupos objetivo; sin embargo, aún persisten percepciones erróneas con respecto a la vivienda de bambú [1][37], lo que podría corregirse con una educación adecuada. Debido a su reputación como un producto sostenible y sus crecientes perspectivas como material de construcción, el bambú tiene una enorme potencialidad en el mercado de la construcción, especialmente en las clases sociales de ingresos bajos a medios [46]. 
E1 uso de bambú como una tecnología de madera laminada [50] puede reemplazar a la madera tradicional en ciertos elementos estructurales. Con un correcto desarrollo de productos, en el que el bambú sea abordado como un nuevo material y no solo como sustituto de la madera, el bambú puede sin duda convertirse en un material alternativo, que permite además el desarrollo de comunidades alrededor de su cultivo y tratamiento.

En muchos casos, los diseñadores trabajan en colaboración con artesanos, creando diferentes productos, cambiando estilos de vida y aplicando nuevas tecnologías para crear un mundo con productos sostenibles de manera ecológicamente compatible como diseño social [51].

\section{Conclusiones.}

La rápida tasa crecimiento, la productividad y la capacidad de los bambúes para sobrevivir y desarrollarse en suelos de baja calidad en comparación con cualquier plantación o sistema forestal hacen que el bambú sea una posibilidad recurso para disminuir el $\mathrm{CO} 2$ atmosférico.

A escala global, el bambú mantiene su huella de carbono negativa; esto continuará mientras el mercado de productos de bambú siga creciendo y se lo pueda utilizar ampliamente como soluciones locales de construcción sostenible.

Aunque las propiedades mecánicas del bambú están por encima de la media, el bambú es muy susceptible al fuego, la humedad e insectos, por lo que se necesita investigar y educar más respecto al diseño y construcción con productos de bambú. Existe evidencia de que el bambú es un material sismorresistente.

La capacidad de secuestrar carbono, la productividad y las nuevas tecnologías combinadas con las técnicas tradicionales representan una fortaleza para situar al bambú como el abanderado en la construcción sostenible en los países en vías de desarrollo.

\section{Referencias Bibliográficas}

[1]M. Añazco Romero and S. Rojas, "Estudio de la cadena desde la producción al consumo del bambú en Ecuador con énfasis en la especie Guadua angustifolia," RED INTERNACIONAL DE BAMBÚ Y RATÁN, INBAR, p. 193, 2015.

[2] Fao, "Global Forest Resources Assessment 2010," 2010.

[3]M. de D. U. y V. MIDUVI, "Estructuras de Guadua (GaK).” 2016.

[4]X. Londoño, "Distribución, morfologia, taxonomia, anatomia, silvicultura y usos de los bambues del nuevo mundo,"Cespedesia, vol. 19, pp. 87-137, 1992.

[5]X. Londoño, G. C. Camayo, N. Riaño, and Y. López, "Characterization of the anatomy of Guadua angustifolia (Poaceae: Bambusoideae) culms," Bamboo Science and Culture, vol. 16, no. 1, pp. 18-31, 2002.

[6]L. A. F. de Moraes, P. Ohayon, and K. Ghavami, "Application of Non-Conventional Materials: Evaluation Criteria for Environmental Conservation in Brazil," Key Engineering Materials, vol. 517, pp. 20-26, 2012. [7]CORPEI, "Bambu ( Caña Guadúa Angus- 
tifolia ) 'Caña Brava'Proyecto Corpei - Cbi

'Expansión De La Oferta Exportable Del Ecuador ," 2003.

[8]Proyecto Corpei-CBI, “Expansión de la oferta exportable del ecuador'|Perfil de producto: Bambú,”pp. 1-25, 2005.

[9] International Network for Bamboo \& Rattan, "Bamboo: A strategic resource for countries to reduce the effects of climate change," Beijing, China, 2014.

[10] A. Jyoti Nath, G. Das, and A. K. Das, "Above ground standing biomass and carbon storage in village bamboos in North East India," Biomass and Bioenergy, vol. 33, no. 9, pp. 1188-1196, 2009.

[11] A. Y. Isagi, T. Kawahara, K. Kamo, and H. Ito, "Net Production and Carbon Cycling in a Bamboo Phyllostachys pubescens Stand,” Plant Ecology, vol. 130, pp. 41-52, 1997.

[12] E. Uchimura, "Ecological studies on cultivation of tropical bamboo forest in the Philippines," Bulletin, Forestry and Forest Products Research Institute, Japan, vol. 22, no. 301, pp. 79-118, 1978.

[13] T. T. Veblen, F. M. Schlegel, and B. Escobar, "Bamboo ( Chusquea Dry-Matter Production of Two Species of in SouthCentral Chile," Journal of Ecology, vol. 68, no. 2, pp. 397-404, 1980.

[14] Y. Isagi, T. Kawahara, and K. Kamo, "Biomass and net produc tion in a bamboo Phyllostachys bambusoides stand," Ecological Research, vol. 8, pp. 123-133, 1993.

[15] E. Z. Escamilla, G. Habert, and E. Wohlmuth, "When CO 2 counts : Sustainability assessment of industrialized bamboo as an alternative for social housing programs in the Philippines," Building and Environment, vol. 103, pp. 44-53, 2016.

[16] R. Monteiro and S. De Carbono, "Ecodesign a eficiência de produtos feitos de Bambu para o sequestro de carbono.pdf," in 10o CONGRESSO BRASILEIRO DE PESQUISA E DESENVOLVIMENTO EM DESIGN, SÃO LUÍS (MA), 2012.

[17] T. Yen and J. Lee, "Comparing aboveground carbon sequestration between moso bamboo ( Phyllostachys heterocycla ) and China fir ( Cunninghamia lanceolata ) forests based on the allometric model," Forest Ecology and Management, vol. 261, no. 6, pp. 995-1002, 2011.

[18] L. Yiping, L. Yanxia, K. Buckingham, G. Henley, and Z. Guomo, "Bamboo and Climate Change Mitigation: a comparative analysis of carbon sequestration,” 2010.

[19] E. Zea Escamilla and G. Habert, "Environmental impacts of bamboo-based construction materials representing global production diversity," Journal of Cleaner Production, vol. 69, pp. 117-127, 2014.

[20] A. D. Ramirez, D. Torres, P. Peña, and J. Duque-Rivera, "Life cycle assessment of greenhouse gas emissions arising from the production of glued and pressed wall panels derived from Guadua Angustifolia Kunth (bamboo) in Ecuador," WIT Transactions on the Built Environment, vol. 142, pp. 447-457, 2014.

[21] J. Vogtländer, P. Van Der Lugt, and H. Brezet, "The sustainability of bamboo products for local and Western European applications. LCAs and land-use," Journal of Cleaner Production, vol. 18, no. 13, pp. 1260-1269, 2010. 
[22] J. J. A. Janssen, "Designing and Building with Bamboo," Beijing, China, 2000.

[23] S. Amada and S. Untao, "Fracture properties of bamboo," Composites Part B:Engineering, vol. 32, no. 5, pp. 451-459, 2001.

[24] L. Villegas, R. Morán, and J. J. García, "A new joint to assemble light structures of bamboo slats," Construction and Building Materials, vol. 98, pp. 61-68, 2015.

[25] I. M. Low, Z. Y. Che, B. A. Latella, and K. S. Sim, "Mechanical and Fracture Properties of Bamboo," Key Engineering Materials, vol. 312, pp. 15-20, 2006.

[26] P. O. Awoyera and E. I. Ugwu, Sustainability and Recycling of Bamboo for Engineering Applications. Elsevier Ltd., 2017.

[27] C. P. Takeuchi and C. E. González, "Resistencia a la compresión paralela a la fibra de la Guadua angustifolia y determinación del módulo de elasticidad," Ingeniería y Universidad, vol. 11, no. 1, pp. 89-103, 2007.

[28] H. F. Archila-Santos, M. P. Ansell, and P. Walker, "Elastic Properties of ThermoHydro-Mechanically Modified Bamboo (Guadua angustifolia Kunth) Measured in Tension," Key Engineering Materials, vol. 600, pp. 111-120, 2014.

[29] K. Lázaro, H. Gonzáles, and A. J. Cardenas, "Propiedades mecánicas del material compuesto elaborado con bambú ( Guadua angustifolia Kunth ) y polipropileno $\mathrm{Me}$ chanical properties of the composite material made with bamboo ( Guadua angustifolia Kunth ) and polypropylene," Revista Mexicana de Ciencias Forestales, vol. 7, no. 38, pp. 95-110, 2016.

[30] C. P. Takeuchi, M. Estrada, and D. L.
Linero, "Determination of Shear Stress in Pull out Tests in Laminated Bamboo Guadua angustifolia,” Key Engineering Materials, vol. 668, pp. 17-22, 2016.

[31] B. Sharma, A. Gatóo, M. Bock, and M. Ramage, "Engineered bamboo for structural applications," Construction and Building Materials, vol. 81, pp. 66-73, 2015.

[32] P. Luna, C. Takeuchi, and E. Cordón, "Mechanical Behavior of Glued Laminated Pressed Bamboo Guadua Using Different Adhesives and Environmental Conditions," Key Engineering Materials, vol. 600, pp. 57-68, 2014.

[33] A. Kumar et al., "Engineered bamboo scrimber: Influence of density on the mechanical and water absorption properties," Construction and Building Materials, vol. 127, pp. 815-827, 2016.

[34] I. Sumardi, K. Ono, and S. Suzuki, "Effect of board density and layer structure on the mechanical properties of bamboo oriented strandboard," Journal of Wood Science, vol. 53, no. 6, pp. 510-515, 2007.

[35] N. Nugroho and N. Ando, "Development of structural composite products made from bamboo II: Fundamental properties of laminated bamboo lumber," Journal of Wood Science, vol. 47, no. 3, pp. 237-242, 2001.

[36] C. P. Takeuchi, M. Estrada, and D. L. Linero, “The Elastic Modulus and Poisson's Ratio of Laminated Bamboo Guadua angustifolia,” Key Engineering Materials, vol. 668, pp. 126-133, 2016.

[37] N. van Drunen, A. Cangás, S. Rojas, and S. Kaminsky, "Reporte post-sismo sobre estructuras de bambú , y recomendaciones 
para la reconstrucción con bambú en la costa Ecuatoriana," Quito, 2016.

S. A. Pardo, F. L. Baez, and N.T. Castellanos, "Connections between a column and its foundation for guadua angustifolia structures under lateral loads," Key Engineering Materials, vol. 668, pp. 227-237, 2016.

[39] A. Harison, A. Agrawal, A. Imam, and A. Harison, "Bamboo as an Alternative to Steel for Green Construction Towards Low Cost Housing," Journal of Environmental Nanotechnology, vol. 6, no. 2, pp. 100-104, 2017.

[40] P. O. Awoyera and A. Adesina, Structural Integrity Assessment of Bamboo for Construction Purposes. Elsevier Ltd., 2017. [41] S. Bhardwaj1 and R. Kumar, "An Alternative to Steel: Bamboo-A review (New Advances), Journal of Engineering Computers \& Applied Sciences(JECAS) ISSN, vol. 3, no. 10, pp. 2319-5606, 2014.

[42] D. M. Nguyen, A. C. Grillet, T. M. H. Diep, C. N. Ha Thuc, and M. Woloszyn, "Hygrothermal properties of bio-insulation building materials based on bamboo fibers and bio-glues," Construction and Building Materials, vol. 155, pp. 852-866, 2017.

[43] Y. Li, J. Yao, R. Li, Z. Zhang, and J. Zhang, "Thermal and energy performance of a steel-bamboo composite wall structure," Energy and Buildings, vol. 156, pp. 225-237, 2017.

[44] A. Gatóo, B. Sharma, M. Bock, H. Mulligan, and M. H. Ramage, "Sustainable structures: bamboo standards and building codes," Proceedings of the Institution of $\mathrm{Ci}^{-}$ vil Engineers - Engineering Sustainability, vol. 167, no. 5, pp. 189-196, 2014.
[45]

E. Salas Delgado, "Actualidad y futuro de la arquitectura de bambú en Colombia. Capítulo 6. Simón Vélez, Símbolo y búsqueda de lo primitivo.,"2006, pp. 125-248.

[46] S. K. Paudel and M. Lobovikov, "Bamboo housing: Market potential for lowincome groups," Journal of Bamboo and Rattan, vol. 2, no. 4, pp. 381-396, 2003.

[47] H. Echezuría, "El Bambú como Recurso Sustentable para Construcción de Viviendas de Bajo Costo,” vol. 21, pp. 52-68, 2018.

[48] G. E. González-Beltrán, "Plybamboo wall-panels for housing Structural Design," 2003.

[49] C. P.Takeuchi, "Design and Construction of Housing with Structural Elements of Bamboo (Guadua) Laminated," Key Engineering Materials, vol. 517, pp. 179-183, 2012.

[50] M. R. Lena, J. F. H. González, J. F. M. Hernández, and S. A. Jiménez, "El tablero de bambú prensado, una nueva propuesta tecnológica," Revista Ingenieria de Construccion, vol. 23, no. 3, pp. 179-186, 2008.

[51] E.-S. Chele, M.-C. Ricardo, P.-M. Ana, and M.-R. Teresa, "Bamboo, from Traditional Crafts to Contemporary Design and Architecture," Procedia - Social and Behavioral Sciences, vol. 51, pp. 777-781, 2012. 